\title{
Affine Connections in Quantum Gravity and New Scalar Fields
}

\author{
Kaushik Ghosh* \\ Vivekananda College (University of Calcutta), 269, \\ Diamond Harbour Road, Kolkata - 700063, India
}

\begin{abstract}
In this manuscript, we will discuss the construction of covariant derivative operator in quantum gravity. We will find it is more perceptive alternative to use affine connections more general than metric compatible connections in quantum gravity. We will demonstrate this using the canonical quantization procedure. This is valid irrespective of the presence and nature of sources. The conventional Palatini and metric-affine formalisms, where the actions are linear in the scalar curvature with metric and affine connections being the the independent variables, are not much suitable to construct a source-free theory of gravity with general affine connections. This is also valid for many minimally coupled interacting theories where sources only couple with metric by using the Levi-Civita connections exclusively. We will discuss potential formalism of affine connections to introduce affine connections more general than metric compatible connections in gravity. We will also discuss possible extensions of the actions for this purpose. General affine connections introduce new fields in gravity besides metric. In this article, we will consider a simple potential formalism with symmetric affine connections and symmetric Ricci tensor. Corresponding affine connections introduce two massless scalar fields. One of these fields contributes a stress-tensor with opposite sign to the sources of Einstein's equation when we state the equation using the Levi-Civita connections. This means we have a massless scalar field with negative stress-tensor in Einstein's equation. This field brings us beyond strict local Minkowski geometries. These scalar fields can be useful to explain inflation and dark energy.
\end{abstract}

Key-words: Affine connections, quantum cosmology, scalar fields, dark energy, inflation

PACS: 98.80.Qc, 98.80.Cq, 95.36.+x, 04.50.Kd, 02.40.-k,

\footnotetext{
* E-mail ghosh_kaushik06@yahoo.co.in
} 


\section{Introduction}

It is now accepted in modern cosmology that the matter and radiation dominated era of cosmic epoch is sandwiched between two periods of cosmic acceleration: inflation and dark energy [1,2,3,4,5,6]. The inflationary scenario is based on some scalar field called inflation. The term dark energy is reserved for an unknown form of energy which not only has not been detected directly, but also does not cluster as ordinary matter does. It is hypothesized to have negative pressure to explain present cosmic acceleration. Besides these, we also need dark matter which does not mediate electromagnetic interactions and are observed by their gravitational effects $[6,7,8,9]$. Present data from different sources, such as the Cosmic Microwave Background Radiation (CMBR) and supernovae surveys, seem to indicate that the energy composition of the universe consists of $20 \%$ dark matter, $76 \%$ dark energy and the rest ordinary baryonic matter. The simplest candidate for dark energy is the cosmological constant $\Lambda$ with constant energy density although we will have to explain its small magnitude $[6,10]$. There are two approaches to explain cosmic acceleration as alternatives to the cosmological constant model. The first is to supplement the source stress-tensor part of Einstein's equation by specific forms of stress-tensor with negative pressure. Among various models, cosmons or quintessence, k-essence and perfect fluid models are mostly studied $[11,12,13]$. The second approach to explain dark energy is to modify the gravitational part of Einstein's equation. Examples are the so-called $f(R)$ gravity [9,14], scalar-tensor theories [15,16] and braneworld models [17,18]. The modified gravity models are more strongly constrained than the modified matter models from astrophysical and cosmological observations. Despite a lot of efforts during the last two decades, it is fair to say that we are yet to explain the origin of the inflation, cosmological constant, dark energy and dark matter. The $\Lambda$-Cold Dark Matter $(\Lambda \mathrm{CDM})$ model can not explain the origin of the above set of fields.

There have been fair amount of works to extend the Einstein-Hilbert action to construct a renormalizable theory of quantum gravity $[19,20]$. It was also found that when quantum corrections are taken into account, the effective low energy gravitational action admits higher order curvature invariants [21,22,23,24]. The gravitational part of Einstein's equation changes when we use such actions. These actions have been useful to construct various modified theories of gravity mentioned above.

In this manuscript, we will try to find if quantum gravity can introduce additional fields besides metric. We will discuss this with reference to the construction of covariant derivative operator in quantum gravity. We can introduce a metric in a spacetime manifold provided it satisfies a few very general topological conditions [25]. To construct covariant differential equations for different tensor fields, we have to introduce additional structures in spacetime. These structures are known as connections. They can be introduced in a differential manifold independent of metric [26,27]. In gravity, we only consider affine connections which we will describe briefly in section:II. In this article we will find it is a perceptive alternative to use affine connections more general than metric compatible affine connections in quantum gravity. We will demonstrate this using the canonical quantization procedure. General affine connections introduce additional fields in the theory that can be useful to explain cosmological observations mentioned before and can introduce new effects. These fields are non-localized similar to dark energy and inflation. In this article, we have considered a simple case with symmetric Ricci tensor. Corresponding affine connections are symmetric and introduce two massless scalar fields. One of these gives a stress tensor with opposite sign to the sources of Einstein's equation in the classical theory when we state the equation using the Levi-Civita connections. This means we have a source with negative stress tensor in the familiar Einstein equation. These scalar fields can be possible candidates for dark energy and inflation. General affine connections break strict local Minkowski structure of spacetime. Global splitting of spacetime into space and time no longer remains exact in such spaces. This is an important issue in quantum gravity $[28,29,30,31]$. Finding the particle interpretations and other possible interactions of the above fields are non-trivial problems without local Minkowski structure. However, experiments suggest that such effects are very small in the present universe and the corresponding scalars mentioned above are also small. Their effects can be observed in large scale phenomena like the present cosmic acceleration. This is also consistent with the smallness of the parameters like the cosmological constant required to explain dark energy in some models mentioned at the beginning. The effects of these fields will be large in the quantum gravity domain. We note that vacuum fluctuations of these fields can also be useful in cosmology. The plan of the paper is then as follows.

We will give a brief description of differential geometry with general affine connections in section:II. This is required to construct a theory of gravity with general affine connections. We will start with a definition of general affine connections $[26,27,28,32,33,34]$. In the rest of this article, we will deal with only affine connections and denote general affine connections by affine connections or connection coefficients. The well-known Levi-Civita connections are a special set of affine connections which obey additional conditions. Affine connections may be asymmetric in the lower indices and need not to be compatible with metric or any symmetric second rank covariant tensor [26]. Affine connections give an additional third rank tensor besides 
metric. In this article, we denote this field by $C^{\alpha}{ }_{\mu \nu}$. Antisymmetric part of this tensor in the covariant indices is half of torsion [26], and give antisymmetric fluctuations in affine connections away from the Levi-Civita connections. Symmetric fluctuations of affine connections off the Levi-Civita connections are described by the symmetric part of $C_{\mu \nu}^{\alpha}$ in the lower indices and this field is not considered in conventional theories of quantum gravity even with sources. In general, this field can be finite when torsion is so. This is evident from Eqs.(25-28). The symmetric part of $C_{\mu \nu}^{\alpha}$ in the lower indices can introduce new symmetric second rank covariant tensors and scalars in gravity besides metric.

In section:III, we will find it is more appropriate to use affine connections more general than metric compatible connections in quantum gravity. We will use the canonical quantization procedure and the Arnowitt-Deser-Misner (ADM) formalism [29] to show this. This is valid irrespective of the presence and nature of sources. This is a general mathematical issue which will be there, in a theory of quantum gravity which is not a quantum field theory in a fixed background, provided some components of metric can be taken as independent variables in a neighborhood of the spacetime manifold. This can be done around any regular point of the spacetime manifold $[35,36,37]$. We will also use the general metric-metric commutators to illustrate this issue [28].

In section:IV, we will discuss the construction of a suitable action, where metric and affine connections are the independent variables, for a quantum theory of gravity. We will first consider the Palatini and metricaffine formalisms $[28,38]$. In these formalisms, the gravitational Lagrangian density is given by the scalar curvature obtained from the corresponding general affine connections. The Palatini action principle leads to metric compatible Levi-Civita connections. This is not suitable to construct quantum gravity where we need to remove the metric compatibility condition even in the source-free theory. The metric-affine theory without sources can only introduce a limited version of non-metricity due to projective invariance. Thus, it is appropriate to extend these formalisms to introduce non-metricity. We will have to extend both the formalisms if we want to have dynamics for $C_{\mu \nu}^{\alpha}$.

We will discuss a potential formalism of affine connections in section: $\mathrm{V}$. By potential formalism we mean a formalism where $C^{\alpha}{ }_{\mu \nu}$ is derived from a tensor of lower rank. This is case with the Levi-Civita connections that are derived from metric. This formalism can introduce finite and dynamic affine connections in the Palatini and metric-affine gravity even when there is no source and introduces a new second rank covariant tensor in the theory. We can also modify the actions by introducing higher order curvature invariants or use theories like Palatini $f(R)$ gravity and metric-affine $f(R)$ gravity to introduce dynamical $C^{\alpha}{ }_{\mu \nu}$. In section:VI we will discuss simple applications of the potential formalism mentioned above.

\section{Affine Connections and Covariant Derivatives}

In this section, we will briefly discuss differential geometry of of curved spaces with general affine connections. We will also mention the generalization of the Einstein-Hilbert action with general affine connections. This formalism is known as the metric-affine theory of gravity [38]. Four properties of ordinary derivatives and transformation rules of tensors under the change of coordinate systems are used to construct covariant derivatives in a general manifold $[26,28]$. Consequently, covariant derivatives in a curved spacetime inherit these properties. We state them in the following:

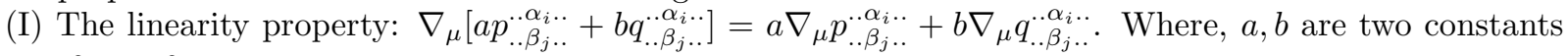
and $p_{. . \beta_{j} . .}^{. \alpha_{i} . .}, q_{. . \beta_{j} . .}^{. \alpha_{i} . .}$ are two well-behaved tensor fields.

(II) For a well-behaved scalar field $f$, and a vector field $t^{\mu}, t(f)=t^{\mu} \nabla_{\mu}(f)$. Here, $t(f)$ denotes directional derivative of the scalar field in the direction of the vector field.

(III) The Leibnitz rule:

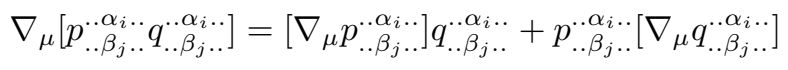

Here, $p_{. . \beta_{j} . . .}^{. . \alpha_{i} . . . \beta_{j} . .}, q_{i} \alpha_{i .}$ are two arbitrary well-behaved tensor fields.

(IV) Commutativity between contraction and covariant derivative:

$$
\nabla_{\mu}\left[C\left(p_{. . . \beta_{j} . . .}^{. \alpha_{i}}\right)\right]=C\left(\nabla_{\mu}\left[p_{. . \beta_{j} . . .}^{. \alpha_{i} .}\right]\right)
$$

Here, $C($ ) denotes contraction operation between upper and lower indices using the Kronecker's delta. These properties and tensorial character of covariant derivatives lead to the following property:

(V) We define torsion tensor through the following relation: 


$$
\left[\nabla_{\mu} \nabla_{\nu}-\nabla_{\nu} \nabla_{\mu}\right] f=-T_{\mu \nu}^{\alpha} \nabla_{\alpha} f
$$

where, $f$ is a well-behaved scalar field. $T_{\mu \nu}^{\alpha}$ is torsion tensor.

We can construct a covariant derivative operator having properties (I - IV):

$$
\nabla_{\mu} A_{\nu}=\ddot{\nabla}_{\mu} A_{\nu}-\Theta_{\mu \nu}^{\alpha} A_{\alpha}
$$

Here, $\Theta_{\mu \nu}^{\alpha}$ are connections. In general relativity, we choose $\ddot{\nabla}_{\mu} \equiv \partial_{\mu}$, where $\partial_{\mu}$ is ordinary partial derivative and we have: $t(f)=t^{\mu} \partial_{\mu}(f)$. Corresponding connections for which $\nabla_{\mu}$ is a covariant derivative operator and satisfy the above conditions are known as affine connections or connection coefficients [26,28]. In this article, we have used the terms affine connections or connection coefficients to denote the most general affine connections. The well known Levi-Civita connections are a special set of affine connections that obey additional conditions to be mentioned below. Tensorial character of covariant derivatives impose the following transformation rule on affine connections:

$$
\bar{\Theta}_{\mu \nu}^{\alpha}=\frac{\partial \bar{x}^{\alpha}}{\partial x^{\lambda}} \frac{\partial x^{\kappa}}{\partial \bar{x}^{\mu}} \frac{\partial x^{\tau}}{\partial \bar{x}^{\nu}} \Theta_{\kappa \tau}^{\lambda}+\frac{\partial \bar{x}^{\alpha}}{\partial x^{\lambda}} \frac{\partial^{2} x^{\lambda}}{\partial \bar{x}^{\mu} \partial \bar{x}^{\nu}}
$$

We can make $\Theta^{\alpha}{ }_{\mu \nu}$ symmetric in the lower indices. Corresponding connections are known as the Chistoffel symbols [28]. We can introduce additional conditions on the Chistoffel symbols. In general relativity, we introduce the Levi-Civita connections through the following metric compatibility conditions [28]:

$$
\nabla_{\mu}\left[g_{\alpha \beta}\right]=0
$$

We have the following expressions for them:

$$
\Theta_{\mu \nu}^{\alpha}=\Gamma_{\mu \nu}^{\alpha}=\frac{1}{2}\left[\partial_{\mu}\left(g_{\kappa \nu}\right)+\partial_{\nu}\left(g_{\mu \kappa}\right)-\partial_{\kappa}\left(g_{\mu \nu}\right)\right] g^{\alpha \kappa}
$$

The above expression shows that the Levi-Civita connections are dependent on partial derivatives of metric and are symmetric in the lower indices. Note that, we can have other solutions of Eq.(6) by introducing additional fields besides metric. We will use such connections below. Torsion is zero when connections are symmetric in the lower indices. Thus, the right hand side of Eq.(3) is zero when we use the LeviCivita connections. The familiar solutions of Einstein's equation in general relativity satisfy the torsion-free condition [28]. In this article, we will show that we have to use affine connections more general than metric compatible connections in quantum gravity.

We next consider the metric-affine theory of gravity. The source-free action is given by [28,38]:

$$
S=\int \sqrt{-g} R \mathbf{e}
$$

Where, $g$ is the determinant of metric, $\sqrt{-g}$ e is the natural volume element associated with metric and $R$ is the scalar curvature. In this formalism, both metric and affine connections are the independent variables. We have the following expression for covariant derivative operator [28]:

$$
\nabla_{\mu} A_{\nu}=\nabla_{\mu}^{\prime} A_{\nu}-C_{\mu \nu}^{\alpha} A_{\alpha}
$$

Here, $C_{\mu \nu}^{\alpha}$ is an arbitrary well-behaved field. It can be symmetric or asymmetric in the lower indices. $\nabla^{\prime}{ }_{\mu}$ is a given covariant derivative which have properties (I - IV) and obey the torsion-free condition. We choose $\nabla_{\mu}^{\prime}$ to be given by the following expression:

$$
\nabla_{\mu}^{\prime} A_{\nu}=\partial_{\mu} A_{\nu}-\Gamma_{\mu \nu}^{\alpha} A_{\alpha}
$$

Here, $\Gamma_{\mu \nu}^{\alpha}$ are the Levi-Civita connections associated with metric $g_{\mu \nu}$. With this choice of $\nabla^{\prime}{ }_{\mu}, C^{\alpha}{ }_{\mu \nu}$ is a third rank tensor. This follows from the transformation properties of affine connections given by Eq.(5) and the 
definition of Levi-Civita connections given by Eq.(7). Note that, $\nabla^{\prime}{ }_{\mu}$ commutes with index raising/lowering operations although $\nabla_{\mu}$ does not. This is another advantage of using Eqs. $(9,10)$.

A restricted version of the metric-affine action mentioned above is obtained if we only consider $C^{\alpha}{ }_{\mu \nu}$ that are symmetric in the lower indices. This is known as the Palatini formalism [28]. In many cases, the symmetric part of affine connections in Eq.(9) can be given by the following expression [26]:

$$
\begin{aligned}
S_{\mu \nu}^{\alpha} & =\Gamma_{\mu \nu}^{\alpha}+\tilde{C}_{\mu \nu}^{\alpha} \\
& =\frac{1}{2}\left[\partial_{\mu}\left(b_{\kappa \nu}\right)+\partial_{\nu}\left(b_{\mu \kappa}\right)-\partial_{\kappa}\left(b_{\mu \nu}\right)\right] \tilde{b}^{\alpha \kappa}
\end{aligned}
$$

Where, $\Gamma_{\mu \nu}^{\alpha}$ are the Levi-Civita connections and given by Eq.(7). $\tilde{C}_{\mu \nu}^{\alpha}$ is the symmetric part of $C^{\alpha}{ }_{\mu \nu}$ in the lower indices. $b_{\mu \nu}$ is a non-singular symmetric covariant tensor and can be expressed as:

$$
b_{\mu \nu}=g_{\mu \nu}+a_{\mu \nu}
$$

The inverse of $b_{\mu \nu}, \tilde{b}^{\mu \nu}$, is a contravariant tensor [26] and can be expressed as $\tilde{b}^{\mu \nu}=g^{\mu \nu}+d^{\mu \nu}$. Note that $\tilde{b}^{\mu \nu}$ is different from $b^{\mu \nu}$ and $S^{\alpha}{ }_{\mu \nu}$ satisfy the compatibility conditions: $b_{\mu \nu \mid \alpha}=0$, where the bar denotes covariant derivative with connections $S_{\mu \nu}^{\alpha}$. We will later find that the Ricci tensor is symmetric when affine connections are exclusively given by Eq.(11). However, Eq.(11) is not valid when the Ricci tensor is not symmetric. The antisymmetric part of $C_{\mu \nu}^{\alpha}$ in the lower indices gives half of torsion tensor [26]. In the metric-affine and Palatini formalisms, metric and respective $C_{\mu \nu}^{\alpha}$ are taken to be the independent variables. The Riemann curvature tensor is now defined by the following expressions, $[26,28,39]$ :

$$
\begin{aligned}
\left(\nabla_{\mu} \nabla_{\nu}-\nabla_{\nu} \nabla_{\mu}\right) A_{\beta}^{\alpha} & =-R_{\mu \nu \kappa}{ }^{\alpha} A^{\kappa}{ }_{\beta}+R_{\mu \nu \beta}{ }^{\kappa} A^{\alpha}{ }_{\kappa}-T^{\kappa}{ }_{\mu \nu} \nabla_{\kappa} A_{\beta}^{\alpha} \\
R_{\mu \nu \alpha}{ }^{\kappa} & =R^{\prime}{ }_{\mu \nu \alpha}{ }^{\kappa}+2 \nabla^{\prime}{ }_{[\nu} C^{\kappa}{ }_{\mu] \alpha}+2\left[C^{\lambda}{ }_{[\mu|\alpha|} C^{\kappa}{ }_{\nu|\lambda|]}\right] \\
T_{\mu \nu}^{\kappa} & =C^{\kappa}{ }_{\mu \nu}-C^{\kappa}{ }_{\nu \mu}
\end{aligned}
$$

Here, $R^{\prime}{ }_{\mu \nu \alpha}{ }^{\kappa}$ is the Riemann curvature tensor associated with the derivative $\nabla_{\mu}^{\prime}$ in Eq.(10), and is given by the familiar expression in terms of ordinary partial derivatives of $\Gamma_{\mu \nu}^{\alpha}[28] . T_{\mu \nu}^{\kappa}$ is torsion tensor. The second equation is always valid when $\Gamma_{\mu \nu}^{\alpha}$ is symmetric in the lower indices. The curvature scalar is obtained by usual contractions and is given by Eq.(22).

\section{Affine Connections in Quantum Gravity}

We now consider quantization of gravity by using the canonical quantization procedure. Canonical quantization is important to find the particle spectrum when we quantize a classical theory. In the canonical quantization of gravity, metric becomes operator on a Hilbert space. We represent such operators by carets. Affine connections present in the covariant derivatives act on the tensor operators and we represent them also by the symbols: $\hat{\Theta}_{\mu \nu}^{\alpha}$. Affine connections will contain components of metric and their spacetime derivatives and also other fields as evident from the previous discussions.

In a Hamiltonian formulation, induced metric on a set of constant time surfaces is used as dynamical variable. The induced metric on a set of constant time surfaces is given by:

$$
h_{\mu \nu}=g_{\mu \nu}+n_{\mu} n_{\nu}
$$

where $n_{\mu}$ is the unit normal to the constant time surfaces. An expression for conjugate momenta is given by Eq.(18). We presently use the symbols $\hat{h}, \hat{\pi}$ to denote the corresponding collection of canonical operators. In general, the Levi-Civita connections contain metric and time derivative of metric components and hence, will depend on the canonical conjugate variables $(\hat{h}, \hat{\pi})$. We now express covariant derivative operator in the following form:

$$
\hat{\nabla}_{\mu}^{\prime} \hat{A}_{\nu}=\left[\partial_{\mu}-\hat{\Gamma}_{\mu \nu}^{\alpha}(\partial \hat{g}, \hat{g})\right] \hat{A}_{\alpha}=\left[\partial_{\mu}-\hat{\Gamma}_{\mu \nu}^{\alpha}(\hat{\pi}, \hat{h})\right] \hat{A}_{\alpha}
$$


Here, $\hat{\Gamma}_{\mu \nu}^{\alpha}$ are operator version of the Levi-Civita connections. We adopt the following operator ordering in connection coefficients. Whenever there appears a product between partial derivatives of metric and metric itself, the partial derivative is kept as the first term and metric is kept as the second term. The ordering of the operators $(\hat{h}, \hat{\pi})$ in $\hat{\Gamma}_{\mu \nu}^{\alpha}$ is given to be the same as that written in the above equation, i.e, $\hat{h}$ is kept as the successor of $\hat{\pi}$.

We next consider the operator: $q^{\mu} \hat{\nabla}_{\mu}^{\prime} q^{\nu}$, where $q^{\mu}$ is a vector field acting as $q^{\mu} \hat{I}$ on the Hilbert space. This operator contains canonical conjugate pairs of variables when we choose affine connections to be given by the Levi-Civita connections. In this case, we will have the following expression:

$$
\left[q^{\mu} \hat{\nabla}_{\mu}^{\prime} q^{\nu}\right]|\Psi\rangle \neq 0
$$

remaining valid in a given state $|\Psi\rangle$ with an arbitrary well-behaved vector field $q^{\mu}$. We will not have a complete set of states for which the expectation value of the operator in the l.h.s is zero with negligible fluctuations for all well-behaved vector fields. This will be valid only in the classical limit, and is a subject similar to the familiar Ehrenfest's theorems in non-relativistic quantum mechanics. Similar discussions will remain valid even if we choose affine connections to be given by the operator versions of Eqs. $(9,10)$. In general, affine connections will contain canonical pairs of variables from metric sector to have proper classical limit of the Levi-Civita connections, and the concept of geodesics will not remain exact for all vector fields in a quantum state. This will also remain valid for parallel transport and the notion of parallel transport is not exact in a quantum theory of gravity. This is expected and indicates that we can use affine connections more general than the metric compatible connections even in free quantum gravity.

We now consider the metric compatibility conditions. The metric compatibility conditions given by Eq.(6) are to be replaced by the operator identity: $\hat{\nabla}_{\mu}^{\prime}\left[\hat{g}_{\alpha \beta}\right] \equiv 0$. The action of $\hat{\nabla}_{\mu}^{\prime}\left[\hat{g}_{\alpha \beta}\right]$ on any state is zero if connection operators are given by the Levi-Civita connection operators and we choose the operator ordering same as that mentioned below Eq.(15). Here, we always keep metric operators as the successors of the partial derivatives of themselves. Thus, the ordering of the different operators in the quantum version of the Levi-Civita connections will be the same as that given in Eq.(7). The same will also remain valid for $\hat{\nabla}_{\mu}^{\prime}\left[\hat{g}_{\alpha \beta}\right]$. Here, $\hat{g}_{\alpha \beta}$ will be kept at the right of the Levi-Civita connections. We also define the contravariant components of metric as: $\hat{g}^{\alpha \kappa} \hat{g}_{\kappa \beta}=\delta_{\beta}^{\alpha}$. This ordering leads to the operator identity: $\hat{\nabla}_{\mu}^{\prime}\left[\hat{g}_{\alpha \beta}\right] \equiv 0$ irrespective of the ordering of $(\hat{h}, \hat{\pi})$ chosen in the partial derivatives of metric components. However, the operator version of metric compatibility conditions need not be consistent with a canonical quantization condition. We will demonstrate this in the following.

As mentioned above, in a Hamiltonian formulation we use induced metric on a set of constant time surfaces as dynamical variable. Thus, $g_{\mu \nu}$ is replaced by: $h_{\mu \nu}=g_{\mu \nu}+n_{\mu} n_{\nu}$. Here, $n_{\mu}$ is given by $(-N, 0,0,0), N$ being the lapse function. The contravariant $n^{\mu}$ is given by: $\frac{1}{N}\left(1,-N^{1},-N^{2},-N^{3}\right)$; where $N^{i}$ are the shift functions. We have: $g_{00}=N_{k} N^{k}-N^{2}, g_{0 i}=N_{i}$ and $N_{i}=g_{i j} N^{j},[29,37]$. The induced metric on the constant time surfaces coincide with the spatial part of $g_{\mu \nu}$ which are expressed as $g_{i j}$. These fields are taken to dynamical variables in general relativity and we have the following Poisson brackets:

$$
\left\{g_{i j}(t, \vec{x}), \pi^{k l}(t, \vec{y})\right\}=\delta_{(i}^{k} \delta_{j)}^{l}[\delta(\vec{x}, \vec{y})]
$$

where, $\vec{x}$ refers to the spatial coordinates and the Poisson bracket is evaluated at equal time. The delta function is defined without recourse to metric. The conjugate momentum is a spatial tensor density and is given by:

$$
\pi^{p l}=-\sqrt{\left|g_{i j}\right|}\left(K^{p l}-K g^{p l}\right)
$$

where $\left|g_{i j}\right|$ is the determinant of the spatial metric, $K_{p l}=-\nabla^{\prime}{ }_{p} n_{l}$ is the extrinsic curvature of the spatial sections, $g^{p l}$ is the inverse of $g_{p l}$ and $K$ is the trace of the extrinsic curvature taken w.r.t $g_{i j}[28,37]$. We can also define the Poisson brackets for the lapse and shift functions although their conjugate momenta vanish giving primary constraints [37]. Thus, in a Hamiltonian formulation with the Einstein-Hilbert action, we have constraints and we can not naively replace the Poisson brackets by commutators when we try to quantize the theory [37]. There are two principal approaches to quantize the theory [36,37]. In the first approach, gauge fixing conditions are introduced to render the complete set of constraints second class [37]. These conditions also determine the lapse and shift functions. We then pick two components of $g_{i j}$ as independent variables and quantize these components using standard commutation relations. We can solve 
the constraints to evaluate other commutators. The second approach is similar to the Gupta-Bleuler method used to quantize electrodynamics and was initiated by Dirac [37,40]. In this approach the classical variables are treated as independent variables and the constraints are imposed on the quantum states. In this case, we can replace the classical Poisson brackets by commutators when we quantize the theory.

We now demonstrate that it is appropriate to extend the Levi-Civita connections and metric compatibility conditions as long as we can regard components of spatial metric on the constant time surfaces as independent physical variables subjected to usual canonical quantization conditions. We will also find that we can not have a Hilbert space on which we can impose the metric compatibility conditions when such quantization conditions remain valid. In the following, we will restrict our attention to a neighborhood around a regular point ' $x$ '. We can extend the neighborhood to the complete spacetime manifold leaving away singularities and other possible irregular points associated with the constraints [36,37]. We pick a component of spatial metric, say $g_{p l}$, as an independent physical variable. We then have the following equal time commutator:

$$
\left[\hat{g}_{p l}(t, \vec{x}), \hat{\pi}^{p l}(t, \vec{y})\right]=i \delta_{(p}^{p} \delta_{l)}^{l}[\delta(\vec{x}, \vec{y})]
$$

where, the point ' $y$ ' belongs to the above mentioned neighborhood of ' $x$ '. There will be another such commutator for the other independent variable. The r.h.s of the commutator is taken to be a distribution that is a spatial tensor density in the spatial coordinates of $\hat{\pi}^{p l}(t, \vec{y})$. The r.h.s will be replaced by different expressions when we replace $\hat{g}_{p l}(t, \vec{x})$ by any dependent component of metric including $g_{0 \mu}$. These terms are determined by the secondary constraints, gauge fixing conditions, definitions of the lapse and shift functions given before and the fundamental commutators given by the above equation. Covariant derivatives give changes in a tensor when we move from one point to a neighbouring point. If the Levi-Civita connections are consistent with the commutators obtained above, corresponding spatial covariant derivatives of both sides of any of the commutators w.r.t the arguments of metric will agree since both sides are equal for all components of metric. We now consider Eq.(19). The action of $\hat{\nabla}_{x k}^{\prime}$ on the r.h.s is same as that on a second rank covariant tensor and will contain spatial partial derivatives of the delta function. It will also contain additional terms dependent on connections and metric that can also explicitly depend on time due to explicit time dependence of the gauge fixing conditions [37]. The later terms are of the form: $-i\left[\hat{\Gamma}_{k p}^{\mu}(t, \vec{x}) \hat{X}_{\mu l}^{p l}+\hat{\Gamma}_{k l}^{\mu}(t, \vec{x}) \hat{X}_{p \mu}^{p l}\right]$, where $i \hat{X}_{\alpha \beta}^{p l}$ is the r.h.s of Eq.(19) when $\hat{g}_{p l}(x)$ is replaced by general $\hat{g}_{\alpha \beta}(x)$. This covariant derivative is not vanishing in general for all values of $\vec{x}$. The left hand side vanishes as can be found from the following expression:

$$
\hat{\nabla}_{x k}^{\prime}\left\{\hat{g}_{p l}(t, \vec{x})\right\} \hat{\pi}^{p l}(t, \vec{y})-\hat{\pi}^{p l}(t, \vec{y}) \hat{\nabla}_{x k}^{\prime}\left\{\hat{g}_{p l}(t, \vec{x})\right\}=0
$$

This follows since we are imposing the operator versions of the metric compatibility conditions. This can also be seen by applying the l.h.s of the above equation to any state, introducing a sum over a complete set of states between the products of the operators and using the fact that the action of $\hat{\nabla}_{\mu}^{\prime}\left[\hat{g}_{\alpha \beta}\right]$ on any state is zero if connection operators are given by the Levi-Civita connection operators with operator ordering chosen below Eq.(16). Thus, the Levi-Civita connections are not consistent with the canonical commutators given by Eq.(19). We will consider other operator ordering in the Levi-Civita symbols in Appendix:A and we will find that similar inconsistency arise in these cases also. Similar situation will remain valid for any point in the manifold where we can introduce constant time surfaces and assume the existence of a metric component as an independent field in a neighborhood around that point. The above inconsistency will also arise with a different choice of constant time surfaces. Thus, there will be a multitude of coordinate systems where we can not use the Levi-Civita connections as connection coefficients if we impose the quantization condition given by Eq.(19). This also indicates that we can not use the Levi-Civita connections as connection coefficients in all coordinate systems that are diffeomorphic to these coordinate systems due to the tensorial character of $C_{\mu \nu}^{\alpha}$.

In Dirac's approach to quantize gravity, all the classical variables are independent and we quantize them accordingly. We can find out $\left[\hat{g}_{0 \beta}(t, \vec{x}), \hat{\pi}^{p l}(t, \vec{y})\right]$ from the definitions of the lapse and shift functions and $\left[\hat{N}^{\lambda}(t, \vec{x}), \hat{\pi}^{p l}(t, \vec{y})\right]=0$, where $N^{0}=N$, [37]. All spatial components $g_{i j}$ satisfy canonical commutation relations given by Eq.(19). We will again have the inconsistency mentioned above when we use the LeviCivita connections. In this case, the action of $\hat{\nabla}_{x k}^{\prime}$ on the r.h.s of Eq.(19) is given by expressions like: $i\left[\partial_{x k} \delta(\vec{x}, \vec{y})-2 \hat{\Gamma}^{0}{ }_{k p}(t, \vec{x}) \hat{N}^{p}(t, \vec{x}) \delta(\vec{x}, \vec{y})-2 \hat{\Gamma}^{p}{ }_{k p}(t, \vec{x}) \delta(\vec{x}, \vec{y})\right]$, where we have taken $p=l$ and there is no sum over the repeated indices. Also, we can not make the Levi-Civita connections consistent with the commutation relation given by Eq.(19) by introducing additional constraints on the physical Hilbert space. If we demand that the action of $\hat{\nabla}_{x k}^{\prime}$ to the r.h.s of Eq.(19) vanishes on the physical Hilbert space, we will have the constraint: 


$$
\left[\partial_{x k} \delta(\vec{x}, \vec{y})-2 \hat{\Gamma}^{0}{ }_{k p}(t, \vec{x}) \hat{N}^{p}(t, \vec{x}) \delta(\vec{x}, \vec{y})-2 \hat{\Gamma}^{p}{ }_{k p}(t, \vec{x}) \delta(\vec{x}, \vec{y})\right]|\Psi\rangle=0
$$

Where, we have again taken $p=l$ and there is no sum over the repeated indices. There will be other similar constraints associated with other commutators. These states also satisfy the secondary constraints. We have used the operator identities $\hat{\nabla}_{\mu}^{\prime} \hat{g}_{\alpha \beta}=0$, and the Levi-Civita connections. All the above conditions will lead to singular expressions involving $\delta(\mathbf{0})$ and the partial derivatives $\delta^{\prime}(\mathbf{0})$ for expectation values of some of the variables: $\hat{g}_{0 \mu}, \hat{g}_{i j}, \hat{N}^{p}$ and $\hat{\Gamma}_{\mu \nu}^{\alpha}$. This is valid for all physical states and is physically undesirable. Lastly, the above problems will arise if we use any set of metric compatible connections.

In the first approach to quantize the theory, it is unlikely that there will exist a set of gauges that is time dependent, render the complete set of constraints second class and also remove the inconsistency mentioned above. It is not possible to remove the inconsistency in the second approach to quantize the theory. Also, it is expected that $\left[\hat{g}_{\alpha \beta}\left(x^{\mu}\right), \hat{g}_{\alpha \beta}\left(y^{\nu}\right)\right]$ will depend on $\left(x^{\mu}, y^{\nu}\right)$ non-trivially with non-vanishing covariant derivatives [28]. We can consider semiclassical theories like quantum fields in curved spaces to assume so. Thus, it is more appropriate to use connections more general than metric compatible connections in quantum gravity. The above discussions are valid irrespective of the presence and nature of sources. We can analyze this issue further in the following way. The Levi-Civita connections and metric compatibility conditions are taken as basic assumptions to calculate the scalar curvature when we use the Einstein-Hilbert action to describe classical and quantum gravity. It is better to discuss quantization and non-metricity using the metric-affine action or Palatini action where $C_{\mu \nu}^{\alpha}$ in Eq.(9) is an independent field. In this article, nonmetricity means $\nabla_{\mu} g_{\alpha \beta} \neq 0$. A quantitative definition is given by Eq.(54). We will discuss the corresponding variational problems and non-metricity in the next section. We will address the issue of $(3+1)$-decomposition of spacetime into space and time in presence of non-metricity below Eq.(55) in section:VI.

\section{Affine Connections and the Lagrangian Formalisms}

In this section, we will discuss the kinematics of the metric-affine and Palatini formalisms. This will help us to identify the degrees of freedom when we use these formalisms that include affine connections as the independent variables. This is important in the quantum theory to impose quantization conditions which require non-metricity. In the Palatini formalism we only consider symmetric $C^{\alpha}{ }_{\mu \nu}$ in Eqs. $(9,10)$. We also consider sources that do not couple with $C^{\alpha}{ }_{\mu \nu}$. Important examples are source free theory and minimally coupled scalar and electromagnetic field in the semiclassical limit of quantum gravity which presently is a locally Lorentz invariant theory of quantum fields in curved spaces. Another important class of examples are many astrophysical systems like the Solar system. In the metric-affine gravity we consider general $C^{\alpha}{ }_{\mu \nu}$ and also sources that couple with $C_{\mu \nu}^{\alpha}$. An example is fermions which couple with torsion through local Lorentz invariance in the semiclassical limit of quantum gravity [41]. However, as we have found in the previous section, we require non-metricity even in source-free quantum gravity. Thus, in the following we first consider the solutions of the variational problem in the metric-affine gravity with sources that do not couple with $C_{\mu \nu}^{\alpha}$. This can include the source-free theory and the Palatini formalism as special cases. We will consider coupling of matter field with $C_{\mu \nu}^{\alpha}$ at the later part of the present section.

In the metric-affine theory, the Ricci tensor is given by: $R_{\mu \nu}=R_{\mu \kappa \nu}{ }^{\kappa}$, where $R_{\mu \kappa \nu}{ }^{\kappa}$ can be obtained from Eq.(13). The Ricci tensor, scalar curvature and metric-affine action are given by the following expressions when we use affine connections given by Eqs. $(9,10)$ :

$$
\begin{aligned}
R_{\mu \alpha} & =R^{\prime}{ }_{\mu \alpha}+2 \nabla^{\prime}{ }_{[\kappa} C^{\kappa}{ }_{\mu] \alpha}+2\left[C_{[\mu|\alpha|}^{\lambda} C^{\kappa}{ }_{\kappa|\lambda|]}\right] \\
R & =R^{\prime}+2 g^{\mu \alpha}\left\{\nabla^{\prime}{ }_{[\kappa} C^{\kappa}{ }_{\mu] \alpha}+\left[C_{[\mu|\alpha|}^{\lambda} C_{\kappa|\lambda|]}^{\kappa}\right]\right\} \\
S & =\int \sqrt{-g} R \mathbf{e}+\kappa_{M} S_{M}\left(\psi, g_{\mu \nu}\right)
\end{aligned}
$$

Where, $R_{\mu \alpha}^{\prime}$ is the Ricci tensor evaluated using the Levi-Civita connections and $R^{\prime}$ is the corresponding scalar curvature. $\psi$ is matter field and the matter field action $S_{M}\left(\psi, g_{\mu \nu}\right)$ does not contain $C_{\mu \nu}^{\alpha} . \kappa_{M}$ is a constant depending on the nature of source [28]. In this article, by matter fields we will mean both matter and gauge fields unless otherwise stated. We now extremize the action given by the last equation w.r.t $C_{\mu \nu}^{\alpha}$. Covariant derivatives of the scalar density $\sqrt{-g}$ are zero when we use the Levi-Civita connections. The second term of the scalar curvature gives a boundary term by the Levi-Civita connections of $\nabla^{\prime}{ }_{\mu}$ and 
Gauss's law form of Stoke's theorem [26,28]. This boundary term vanishes when $C_{\mu \nu}^{\alpha}$ is held fixed at the boundary. We then have the following equation as the solution of variational problem when $C_{\mu \nu}^{\alpha}$ is held fixed at the boundary:

$$
C^{\kappa}{ }_{\kappa \lambda} g^{\mu \alpha}+C^{\alpha \kappa} \delta_{\lambda}^{\mu}-C_{\lambda}^{\mu \alpha}-C_{\lambda}^{\alpha \mu}=0
$$

There is no contribution from the source fields in the present case. This is an algebraic equation giving constraints on $C_{\mu \nu}^{\alpha}$. We obtain the following equation when we extremize the action w.r.t $g_{\mu \nu}$ :

$$
\begin{aligned}
\mathcal{G}_{(\mu \alpha)} & =\mathcal{R}_{(\mu \alpha)}-\frac{1}{2} \mathcal{R} g_{\mu \alpha}=8 \pi P_{\mu \alpha} \\
\mathcal{R}_{\mu \alpha} & =R^{\prime}{ }_{\mu \alpha}+2\left[C^{\lambda}{ }_{[\mu|\alpha|} C^{\kappa}{ }_{\kappa|\lambda|]}\right] \\
\mathcal{R} & =g^{\mu \alpha} \mathcal{R}_{\mu \alpha}
\end{aligned}
$$

Where, $\mathcal{R}_{\mu \nu}$ and $\mathcal{G}_{\mu \nu}$ are the modified Ricci tensor and modified Einstein tensor respectively. They coincide with the Ricci tensor and Einstein tensor when $C_{\mu \nu}^{\alpha}$ is absent. $P_{\mu \alpha}$ is matter field stress tensor which is related to the variational derivative of matter field action w.r.t $g^{\mu \alpha}$ by: $8 \pi P_{\mu \alpha}=-\frac{\kappa_{M}}{\sqrt{-g}} \frac{\delta S_{M}}{\delta g^{\mu \alpha}}$. Here $\delta$ denotes functional derivatives and $P_{\mu \alpha}$ is symmetric. Again, the second term of the curvature scalar does not contribute to Einstein's equation by the Levi-Civita connections of $\nabla^{\prime}{ }_{\mu}$ and the Gauss's law form of Stoke's theorem when metric is held fixed at the boundary. The first order change in $R_{\mu \alpha}^{\prime}$ due to change in metric is given by: $\nabla_{[\kappa}^{\prime} \delta \Gamma^{\kappa}{ }_{\mu] \alpha}$, where $\delta \Gamma_{\mu \alpha}^{\kappa}$ is the change in Levi-Civita connection due to change in metric. This term does not contribute to the equation of motion by the Stoke's theorem. Here, we have assumed that metric and its first order derivatives are held fixed at the boundary [28].

We now construct the solutions of Eq.(23). A contraction over $(\lambda, \mu)$ leads to the following equation:

$$
H_{\kappa}^{\kappa}{ }^{\alpha}+\frac{3}{2} \tilde{C}_{\kappa}^{\alpha \kappa}=0
$$

An alternate contraction over $(\alpha, \mu)$ leads to the following equation:

$$
4 H_{\kappa \lambda}^{\kappa}+2 \tilde{C}_{\kappa \lambda}^{\kappa}+\tilde{C}_{\lambda \kappa}^{\kappa}=0
$$

Here, $\tilde{C}_{\mu \nu}^{\alpha}$ is the symmetric part of $C^{\alpha}{ }_{\mu \nu}$ and $H_{\mu \nu}^{\alpha}=\frac{1}{2}\left(C_{\mu \nu}^{\alpha}-C_{\nu \mu}^{\alpha}\right)=\frac{1}{2} T_{\mu \nu}^{\alpha}$, is half of torsion tensor. These two equations give the following equations from Eq.(23):

$$
H_{\kappa \lambda}^{\kappa} g_{\mu \alpha}+H_{\kappa(\alpha}^{\kappa} g_{\mu) \lambda}+3 \tilde{C}_{(\mu \alpha) \lambda}=0
$$

and

$$
H_{\kappa[\alpha}^{\kappa} g_{\mu] \lambda}+3 H_{[\mu \alpha] \lambda}=0
$$

Eqs. $(27,28)$ give a set of homogeneous equations for $C^{\alpha}{ }_{\mu \nu}$ that can not determine $C^{\alpha}{ }_{\mu \nu}$ uniquely. We have four-fold ambiguities associated with the projective transformation given by: $C^{\alpha}{ }_{\mu \nu} \rightarrow C^{\alpha}{ }_{\mu \nu}+\delta_{\nu}^{\alpha} \xi_{\mu}$, where $\xi_{\mu}$ is a regular covariant vector field [38]. This is a symmetry of the gravitational part of metric-affine action and also of Eq.(23). Projective transformation can introduce a limited version of non-metricity. This no longer remains valid for the Palatini formalism where we only consider symmetric $C^{\alpha}{ }_{\mu \nu}$. Eqs. $(27,28)$ give us the Levi-Civita connections when we set $H_{\kappa \mu}^{\kappa}=0$ which fixes $\xi_{\mu}=0$. Projective invariance need not to be pertinent when we add sources in the metric-affine theory. Without any dynamic equation for itself, $\xi_{\mu}$ can not produce non-trivial vacuum effects. Thus we will no longer consider projective transformation in the following. We now consider the case when $C^{\alpha}{ }_{\mu \nu}$ is purely antisymmetric in the lower indices. In this case, we have the following equations:

$$
H_{[\mu \alpha] \lambda}=0
$$


This gives vanishing solutions. With purely symmetric connections in the Palatini formalism, Eq.(27) also gives vanishing solutions.

The above formalism will give non-vanishing solutions for $C_{\mu \nu}^{\alpha}$ in presence of sources that couple with $C_{\mu \nu}^{\alpha}$. This is the case in the metric-affine gravity with fermions if we consider locally Lorentz invariant theory of quantum fields in curved spaces. Eq.(23) is replaced by the following expression:

$$
\begin{aligned}
C_{\kappa \lambda}^{\kappa} g^{\mu \alpha}+C^{\alpha \kappa} \delta_{\lambda}^{\mu}-C_{\lambda}^{\mu \alpha}-C_{\lambda}^{\alpha \mu} & =\Delta_{\lambda}{ }_{\lambda}^{\mu \alpha} \\
\Delta_{\lambda}{ }^{\mu \alpha} & =-\kappa_{M} \frac{\delta S_{M}\left(\psi, g_{\mu \nu}, C_{\mu \nu}^{\lambda}\right)}{\delta C_{\mu \nu}^{\lambda}}
\end{aligned}
$$

Where, $\Delta_{\lambda}{ }^{\mu \alpha}$ is known as the hypermomentum $[32,38]$. However, matter field Lagrangians are usually polynomials in first order covariant derivatives. In addition, gauge fields do not couple with $C_{\mu \nu}^{\alpha}$ to express the corresponding field-strength tensors as gauge-covariant curls [41]. We will illustrate this briefly in section:VI. Thus, the above equation remains an algebraic constraint. Hence, it is appropriate to extend the metric-affine and Palatini formalisms in quantum gravity to have nontrivial dynamics for $C_{\mu \nu}^{\alpha}$. In the next section, we will discuss a few theories as possible candidates for a theory of gravity with dynamic $C_{\mu \nu}^{\lambda}$.

In the metric-affine theory with sources, we have the scope to have finite $\tilde{C}_{\mu \nu}^{\alpha}$ when torsion is finite unless we have a special source such that: $H_{\kappa \lambda}^{\kappa}=0$. The antisymmetric part of the Ricci tensor is given by the following expression:

$$
R_{[\mu \alpha]}=\nabla_{\kappa}^{\prime} H_{\mu \alpha}^{\kappa}-\nabla_{[\mu}^{\prime} C_{|\kappa| \alpha]}^{\kappa}+H_{\mu \alpha}^{\lambda} C_{\kappa \lambda}^{\kappa}+\left(\tilde{C}_{\kappa \alpha}^{\lambda} H_{\lambda \mu}^{\kappa}-\tilde{C}_{\kappa \mu}^{\lambda} H_{\lambda \alpha}^{\kappa}\right)
$$

Where, $\nabla^{\prime}{ }_{\kappa}$ is evaluated using the Levi-Civita part of complete connections. Note that, $R_{\mu \alpha}^{\prime}$ is symmetric because: $\Gamma_{\kappa \alpha}^{\kappa}=\partial_{\alpha}(\ln \sqrt{\mid} g \mid)$, where $|g|$ is the absolute value of the metric determinant. Thus, in the metricaffine formalism, the Ricci tensor is not symmetric in general in presence of sources. A purely symmetric Ricci tensor will impose 6 additional constraints on the sources. The same is valid for the modified Ricci tensor. In this case, the derivative terms will be absent. This is also important to construct a semiclassical theory of fermions in a curved spacetime that can be derived from a variational principle.

The following discussions in this paragraph are valid when $(3+1)$-splitting is possible. This is a nontrivial issue when we use general affine connection and will be discussed below Eq.(55). In the metric-affine formalism discussed above, we can quantize the theory by considering $\left(g_{\mu \nu}, C_{\mu \nu}^{\alpha}\right)$ as the complete set of variables. Conjugate momenta of $C_{\mu \nu}^{\alpha}$ vanish if we discard the four-divergences in the Lagrangian density. Affine connections do not spoil the diffeomorphism invariance of the theory and the constraints will include three sets: (i) four secondary constraints coming from the non-dynamical nature of the lapse and shift functions and four gauge fixing conditions; (ii) 64 primary constrains: $\pi_{\alpha}^{\mu \nu}=0$; (iii) 64 secondary constraints given by Eq.(23) which are equivalent to: $C_{\mu \nu}^{\alpha}=0$. The complete set of constraints are second class with suitable gauge fixing conditions and we can try to impose quantization conditions similar to Eq.(19). We will again have inconsistencies similar to that discussed in the previous section if we use the Levi-Civita connections. With $C_{\mu \nu}^{\alpha}=0$ being the unique solutions of Eq.(23), we will have to extend the standard Palatini formalism to quantize generalized free gravity. This will also lead to general affine connections. These aspects are also consistent with the discussions given below Eq.(29).

We conclude this section with a few comments on commutators. We consider the field-field commutators for independent variables at two general space time points:

$$
\left[\hat{\phi}_{M}\left(x^{\mu}\right), \hat{\phi}_{M}\left(y^{\nu}\right)\right]=i D_{M, M}\left(x^{\mu}, y^{\nu}, \hat{\phi}_{P}\right)
$$

Where, $\hat{\phi}_{M}$ is an independent field operator from the complete set of fields that include metric and $C^{\alpha}{ }_{\mu \nu}$. We will use the mixed tensor $C_{\mu \nu}^{\alpha}$ to find the commutators since it can give covariant derivatives of mixed tensors. $D_{M, M}$ will depend on the type of field and should be consistent with connection coefficients of the theory. In the case of gravity, general commutators: $\left[\hat{g}_{\alpha \beta}\left(x^{\mu}\right), \hat{g}_{\alpha \beta}\left(y^{\nu}\right)\right]$ will depend on $\left(x^{\mu}, y^{\nu}\right)$ nontrivially, and it is appropriate to use connections more general than metric compatible connections in the quantum theory. These metric-metric commutators will satisfy equations of the form:

$$
\hat{\nabla}_{x \tau} D_{\alpha \beta, \alpha \beta}=2 i\left[\hat{C}_{(\alpha|\tau| \beta)}\left(x^{\mu}\right), \hat{g}_{\alpha \beta}\left(y^{\nu}\right)\right]
$$

The equal time commutators are supported at $\vec{x}=\vec{y}$ in locally or globally Lorentz invariant quantum field theories. This may not remain strictly valid in quantum gravity, in particular when $(3+1)$-decomposition is not possible. 


\section{Affine Connections and the Extended Lagrangian Formalisms}

The variational problems discussed in the previous section give two sectors of equations. The first sector is obtained by varying the action w.r.t $C_{\mu \nu}^{\alpha}$ and is given by Eqs.(23,30). Eq.(23) is homogeneous and contains no time derivatives. It acts like a constraint. We obtain Eq.(30) when we include suitable sources. However, Eq.(30) is also an algebraic equation for the known sources which are presently fermions coupled with torsion and we will have to extend these formalisms to introduce dynamic $C_{\mu \nu}^{\alpha}$. We will also have to extend the metric-affine formalism developed for quantum field theory in curved spaces where metricity is a constraint to have locally Minkowskian structure of the background spacetime [38,41]. We first try to extend the formalism to construct a quantum theory with finite and dynamic $C_{\mu \nu}^{\alpha}$. Here, we extend the formalisms in two alternate ways. The equations obtained are differential equations with time derivatives.

We can construct a theory by using the potential formalism. By potential formalism we mean a formalism where $C_{\mu \nu}^{\alpha}$ is derived from a tensor of lower rank. This is case with the Levi-Civita connections that are derived from metric. We will then have a set of differential equations including time derivatives in place of Eqs. $(23,30)$ and we can introduce non-metricity even in the source-free theory by using nontrivial solutions of the homogeneous differential equations. We can use Eqs.(11,12) to define symmetric connections, where $a_{\mu \nu}, b_{\mu \nu}$ are symmetric. Non-metricity is ensured by: $a_{\mu \nu} \neq k g_{\mu \nu}$, where $k$ is a constant which can be zero. This is a mild constraint, the otherwise of which gives the Levi-civita connections. The Ricci tensor is symmetric in this case. This is obvious from $\nabla_{\mu}^{\prime} \equiv \partial_{\mu}$ and Eq.(31). We have:

$$
C_{\kappa \alpha}^{\kappa}=S_{\kappa \alpha}^{\kappa}=\partial_{\alpha}(\ln \sqrt{|b|}) ; H_{\alpha \beta}^{\kappa}=0
$$

Where $b$ is the determinant of $b_{\mu \nu}$ given in Eqs.(11,12). We have: $\partial_{[\mu} \partial_{\alpha]}(\ln \sqrt{\mid}|b|)=0$ and $R_{[\mu \nu]}$ vanishes by Eq.(31). Thus, it is appropriate to use $a_{\mu \nu}$ to describe the connections when the Ricci tensor is symmetric. Note that $\sqrt{|b|}$ is a scalar density. In the next section, we will consider two simple cases that give nonmetricity and that can be useful to explain cosmological accelerations.

We now discuss another model with zero torsion. Thus, we are considering the Palatini formalism. We asuume that $\tilde{C}_{\mu \nu}^{\alpha}$ is given by:

$$
\tilde{C}_{\mu \nu}^{\alpha}=\nabla^{\prime \alpha} q_{\mu \nu}
$$

Here $q_{\mu \nu}$ is a symmetric tensor and $\nabla^{\prime}{ }_{\alpha}$ uses the Levi-Civita connections given by Eqs. $(9,10)$. This can be useful when the Ricci tensor is not symmetric. We put this expression in the Palatini action given by Eqs. $(8,22)$. We then take variational derivatives $w . r . t q_{\mu \nu}$. In this case, the action contains a second order derivative which does not contribute to the variational derivative by the Levi-Civita connections of $\nabla^{\prime}{ }_{\mu}$ and the Gauss's law form of Stoke's theorem [26]. We have the following equation:

$$
2 \nabla^{\prime \kappa} \nabla^{\prime(\mu} q_{\kappa}^{\alpha)}-g^{\mu \alpha} \nabla_{\lambda}^{\prime} \nabla^{\prime \kappa} q_{\kappa}^{\lambda}-\nabla^{\prime \mu} \nabla^{\prime \alpha} q=0
$$

Where, $g_{\mu \nu}$ is a solution of the metric sector Einstein's equation, $\nabla^{\prime}{ }_{\lambda}$ is the corresponding metric compatible covariant derivative that uses the Levi-Civita connections and $q$ is the trace of $q_{\mu \nu}$. All the above equations are dynamical and all conjugate momenta are finite. Moreover, time derivative of all components of $q_{\kappa}^{\lambda}$ are present in the complete set of equations. In this case, the modified Ricci tensor is symmetric and the Ricci tensor can be made symmetric by imposing the constraint: $\nabla_{[\mu}^{\prime} \tilde{C}_{|\kappa| \alpha]}^{\kappa}=0$. This leads us to introduce a scalar field:

$$
\nabla^{\prime \kappa} q_{\kappa \alpha}=-\nabla_{\alpha}^{\prime} \zeta
$$

Eq.(36) gives us the following relation between the trace of $q_{\mu \nu}$ and $\zeta$ :

$$
\nabla_{\mu}^{\prime} \nabla^{\prime \mu}(q)=2 \nabla_{\mu}^{\prime} \nabla^{\prime \mu}(\zeta)
$$

There will be a non-holonomic constraint to ensure nonmetricity: $\nabla^{\prime(\mu} q_{\kappa}^{\alpha)} \neq 0$. The trace of $\nabla^{\prime(\mu} q_{\kappa}^{\alpha)}$ is $-\nabla^{\prime}{ }_{\kappa} \zeta$, when the corresponding Ricci tensor is symmetric. 
We can also construct a special model with: $H_{\mu \nu}^{\alpha}=\nabla^{\prime \alpha} f_{\mu \nu}$, where $f_{\mu \nu}$ is an antisymmetric tensor, and include both $q_{\mu \nu}$ and $f_{\mu \nu}$. A detail potential formalism of torsion is discussed in [42]. Here we have the following set of equations for $q_{\mu \nu}$ and $f_{\mu \nu}$ :

$$
\begin{aligned}
& 2 \nabla^{\prime \kappa} \nabla^{\prime(\mu} q_{\kappa}^{\alpha)}-g^{\mu \alpha} \nabla_{\lambda}^{\prime} \nabla^{\prime \kappa} q_{\kappa}^{\lambda}-\nabla^{\prime(\mu} \nabla^{\prime \alpha)} q+g^{\mu \alpha} \nabla^{\prime \lambda} \nabla^{\prime \kappa} f_{\lambda \kappa}=0 \\
& \nabla^{\prime \lambda}\left\{\nabla^{\prime[\mu} f_{\lambda}^{\alpha]}\right\}-\nabla^{\prime[\mu} \nabla^{\prime \alpha]} q=0
\end{aligned}
$$

This is a set of coupled differential equations which are dynamical in nature. The last term in both the equations give coupling between $q_{\mu \nu}$ and $f_{\mu \nu}$ and vanishes when torsion is vanishing. We again find that in the metric-affine theory we have finite $q_{\mu \nu}$ when $f_{\mu \nu}$ is finite. Non-metricity need not to preserve local Minkowskian structure of spacetime [38], and the particle interpretations of these fields are not obvious. These fields, although massless, can be important in inflation driven by scalar as well as higher spin fields $[43,44,45,46]$. We will later discuss the dynamics of this model. If required, we can also use other potentials for torsion [42].

Alternatively, we can use different actions to construct the dynamics of $C_{\mu \nu}^{\alpha}$ field itself. We can include higher order curvature scalars like $R^{\mu \nu} R_{\mu \nu}$ in the Einstein-Hilbert Lagrangian with the Riemann curvature tensor evaluated using finite $C^{\alpha}{ }_{\mu \nu}$ as given by Eq.(13). This is interesting if we consider the renormalizability of quantum gravity. We can also use extended theories of gravity like the Palatini $f(R)$ gravity or metricaffine $f(R)$ gravity. One can look at $[6,8]$ for reviews on this topic. When we use the curvature scalar given by Eq.(22) in a metric-affine $f(R)$ gravity, we can no longer neglect the total divergence middle term: $2 g^{\mu \alpha} \nabla_{[\kappa}^{\prime} C_{\mu] \alpha}^{\kappa}$. It will couple with other terms of $R_{\mu \nu}$ and we will have differential equations for $C_{\mu \alpha}^{\kappa}$ in general. This can give dynamic on shell $C_{\mu \alpha}^{\kappa}$. We will have to ensure non-metricity. We will again have finite $\tilde{C}_{\mu \nu}^{\alpha}$ in general when $H_{\mu \nu}^{\alpha}$ is finite. We can also use the potential formalism in such theories. A relevant formulation is the dynamical theory of metric compatible torsion (contorsion) discussed by a few authors with different actions $[47,48]$. These papers introduce metric compatible torsion in an effective action of quantum gravity obtained from the Einstein-Hilbert action and discuss propagators for the corresponding particles. Corresponding potential theory of torsion is ghost free in this formalism [47]. For our purpose, it is appropriate to extend the Einstein-Hilbert-Palatini action at the classical level. We can also use theories like string theory.

\section{Applications in Cosmology}

In this section, we will consider applications of the potential formalism of the Palatini theory discussed previously to introduce finite on shell non-metricity. Corresponding $C_{\mu \nu}^{\alpha}$ are symmetric in the lower indices and the sources do not couple with these fields. We have mentioned some examples in sections:II,V. We also consider symmetric Ricci tensors only. This is the most relevant case when we consider semiclassical and classical limits of quantum gravity. This example will introduce two massless scalar fields. One of them will contribute a negative stress tensor to Einstein's equation in the semiclassical or classical theories. We will use the geometrized units in the following where, $G=c=1$.

In section:II, we have mentioned that we can introduce non-metricity by defining the affine connections to be compatible with a symmetric covariant field: $b_{\mu \nu}=g_{\mu \nu}+a_{\mu \nu} ; a_{\mu \nu} \neq k g_{\mu \nu}$, where $k$ is a constant including zero. In the last section we have found that such a set of affine connections give a symmetric Ricci tensor. We now break $a_{\mu \nu}$ into a trace and a traceless part:

$$
a_{\mu \nu}(x)=\Phi(x) g_{\mu \nu}+\bar{a}_{\mu \nu}(x) ; \quad \Phi(x)=\frac{a(x)}{4}
$$

Where, $\Phi$ is a scalar field, $a(x)$ is the trace of $a_{\mu \nu}$ and $\bar{a}_{\mu \nu}$ is trace-free. We can express corresponding $\tilde{C}_{\mu \nu}^{\alpha}$ in the following way:

$$
\tilde{C}_{\mu \nu}^{\alpha}=\delta_{(\mu}^{\alpha} \nabla_{\nu)}^{\prime}[\ln (1+\Phi)]-\frac{1}{2} g_{\mu \nu} \nabla^{\prime \alpha}[\ln (1+\Phi)]+D_{\mu \nu}^{\alpha}
$$

The first two terms in the r.h.s gives the contribution of the trace part of $b_{\mu \nu}$ given by: $\left(g_{\mu \nu}+\Phi(x) g_{\mu \nu}\right)$, [28]. $D_{\mu \nu}^{\alpha}$ can be expressed in terms of $g_{\mu \nu}, a_{\mu \nu}$ and their derivatives. A further contraction of the third term in the covaraiant indices leads to the following expression for a general $a_{\mu \nu}$ : 


$$
\begin{aligned}
\tilde{C}_{\mu \nu}^{\alpha} & =\delta_{(\mu}^{\alpha} \nabla_{\nu)}^{\prime}[\ln (1+\Phi)]-\frac{1}{2} g_{\mu \nu} \nabla^{\prime \alpha}[\ln (1+\Phi)]+g_{\mu \nu} H^{\alpha}+E_{\mu \nu}^{\alpha} \\
& =\delta_{(\mu}^{\alpha} \nabla_{\nu)}^{\prime}[\ln (1+\Phi)]-\frac{1}{2} g_{\mu \nu} \nabla^{\prime \alpha}[\ln (1+\Phi)]+g_{\mu \nu} \nabla^{\prime \alpha} \Psi+g_{\mu \nu} B^{\alpha}+E_{\mu \nu}^{\alpha}
\end{aligned}
$$

where $\Phi$ gives the trace-scalar of $a_{\mu \nu}$ and $E^{\alpha}{ }_{\mu \nu}$ is traceless in the lower indices. $\Psi$ is a scalar field with $\nabla^{\prime 2} \Psi=\nabla^{\prime}{ }_{\mu} H^{\mu}$. $B^{\alpha}$ is a vector field with: $\nabla^{\prime}{ }_{\alpha} B^{\alpha}=0$, and is suitable to describe a spin one boson. However, we also have: $\nabla^{\prime}{ }_{\mu} B_{\nu]}=0$, which follows from the symmetry of the Ricci tensor that remains valid with a symmetric $a_{\mu \nu}$. In the following we will take $B^{\mu}=0 . \Psi$ is expected. In the Minkowski space, we have a spin zero boson associated with $\bar{a}_{\mu \nu}$ in addition to a spin one boson and a spin two boson. We will later briefly discuss $E^{\alpha}{ }_{\mu \nu}$ which is expected to describe a spin two boson.

We now consider the simplest cases of Eq.(42). We first consider the case where the traceless part of $a_{\mu \nu}$ vanish and which introduces a scalar field only:

$$
b_{\mu \nu}=g_{\mu \nu}+\Phi(x) g_{\mu \nu}=\chi(x) g_{\mu \nu}
$$

We use affine connections that are compatible with $b_{\mu \nu}$ which is conformal to metric. In this case, $\tilde{C}_{\mu \nu}^{\alpha}$ and the modified curvature scalar are given by the following expressions [28]:

$$
\begin{aligned}
\tilde{C}_{\mu \nu}^{\alpha} & =\delta_{(\mu}^{\alpha} \nabla_{\nu)}^{\prime}[\ln (1+\Phi)]-\frac{1}{2} g_{\mu \nu} \nabla^{\prime \alpha}[\ln (1+\Phi)] \\
\mathcal{R} & =R^{\prime}-\frac{3}{2} \frac{1}{(1+\Phi)^{2}}\left[\nabla^{\prime}(1+\Phi)\right]^{2}=R^{\prime}-\frac{3}{2}\left[\nabla^{\prime} \ln (\chi)\right]^{2}
\end{aligned}
$$

Here $\left(\nabla^{\prime} \Phi\right)^{2}=\left(\nabla_{\mu}^{\prime} \Phi\right)\left(\nabla^{\prime \mu} \Phi\right)$, is the norm of the gradient of $\Phi$ and the primed quantities are evaluated using the Levi-Civita connections. For small $\Phi(<<1)$, we have the following modification of the r.h.s of Einstein's equation where covariant derivatives are evaluated using the Levi-Civita connections [28]:

$$
\begin{aligned}
\frac{1}{\chi} \nabla_{\kappa}^{\prime} \nabla^{\prime \kappa} \ln (\chi) & =\frac{1}{\chi^{2}}\left[\nabla^{\prime}{ }_{\kappa} \nabla^{\prime \kappa} \chi-\frac{1}{\chi}\left(\nabla^{\prime} \chi\right)^{2}\right]=0 \approx \nabla^{\prime}{ }_{\kappa} \nabla^{\prime \kappa} \Phi-\left(\nabla^{\prime} \Phi\right)^{2} \\
G^{\prime}{ }_{\mu \alpha} & =8 \pi P^{\prime}{ }_{\mu \alpha}+\frac{3}{2 \chi^{2}}\left[\left(\nabla^{\prime}{ }_{\mu} \chi\right)\left(\nabla^{\prime}{ }_{\alpha} \chi\right)-\frac{1}{2} g_{\mu \alpha}\left(\nabla^{\prime} \chi\right)^{2}\right] \\
& \approx 8 \pi P^{\prime}{ }_{\mu \alpha}+\frac{3}{2}\left[\left(\nabla^{\prime}{ }_{\mu} \Phi\right)\left(\nabla^{\prime}{ }_{\alpha} \Phi\right)-\frac{1}{2} g_{\mu \alpha}\left(\nabla^{\prime} \Phi\right)^{2}\right] \\
& =8 \pi\left[P_{\mu \alpha}^{\prime}+\frac{3}{16 \pi} P_{\mu \alpha}^{\prime}{ }_{\mu \alpha}(\Phi)\right]
\end{aligned}
$$

Where, we have considered terms upto second order in $\Phi . P^{\prime}{ }_{\mu \alpha}$ is the stress tensor of ordinary matter. $P^{\prime}{ }_{\mu \alpha}(\Phi)$ is the stress tensor of an ordinary masless scalar field. Both $\chi$ and $\Phi$ are massless. The exact equations are consistent with: $\nabla^{\prime \alpha} G^{\prime}{ }_{\mu \alpha}=0$. We find that the scalar field $\Phi$ behaves like a massless scalar field with its stress tensor coming as a source term in Einsrein's equation. This field can be useful to explain inflation. We can modify the metric-affine action by adding potential term for $\chi$ or $\Phi$. For non-metricity, we have the following condition:

$$
\nabla_{\mu} g_{\alpha \beta}=-g_{\alpha \beta} \nabla_{\mu}^{\prime}[\ln (1+\Phi)] \neq 0
$$

We will later discuss the significance. In the present case, matter fields do not couple with $\Phi$. The only observable effect of $\Phi$ is to produce a massless scalar field stress tensor in Einstein's equation.

We now consider the other case where only $\Psi$ is finite:

$$
\tilde{C}_{\mu \nu}^{\alpha}=g_{\mu \nu} \nabla^{\prime \alpha} \Psi
$$

We have the following expressions for different quantities: 


$$
\begin{aligned}
\mathcal{R}_{\mu \alpha} & =R^{\prime}{ }_{\mu \alpha}+g_{\mu \alpha}\left(\nabla^{\prime} \Psi\right)^{2}-\left(\nabla_{\mu}^{\prime} \Psi\right)\left(\nabla^{\prime}{ }_{\alpha} \Psi\right) \\
\mathcal{R} & =R^{\prime}+3\left(\nabla^{\prime} \Psi\right)^{2}
\end{aligned}
$$

We now solve the corresponding extremization problem and obtain the following set of equations:

$$
\begin{aligned}
\nabla_{\kappa}^{\prime}{ }_{\kappa}{ }^{\prime \kappa} \Psi & =0 \\
G_{\mu \alpha}^{\prime} & =8 \pi P^{\prime}{ }_{\mu \alpha}-3\left[\left(\nabla_{\mu}^{\prime} \Psi\right)\left(\nabla_{\alpha}^{\prime} \Psi\right)-\frac{1}{2} g_{\mu \alpha}\left(\nabla^{\prime} \Psi\right)^{2}\right] \\
& =8 \pi\left[P^{\prime}{ }_{\mu \alpha}-\frac{3}{8 \pi} P_{\mu \alpha}^{\prime}(\Psi)\right]
\end{aligned}
$$

Where, $P^{\prime}{ }_{\mu \alpha}$ is the stress-tensor of ordinary sources and $P^{\prime}{ }_{\mu \alpha}(\Psi)$ is the stress-tensor of an ordinary massless scalar field. This stress tensor satisfy all the energy and pressure conditions that the stress-tensor of a massless scalar field satisfies but it comes with an opposite sign in the r.h.s of Einstein's equation, when we state the equation using the Levi-Civita connections. Thus, the effect of $\Psi$ introduced to generalize the LeviCivita connections, is to contribute a negative massless scalar field stress tensor to the sources of the Einstein equation obtained from the Einstein-Hilbert action formalism. This gives us an alternate way to explain effects that dark energy is proposed for. Negative stress-tensor is also important in Hoyle-Narlikar theory of gravity and also in wormhole and warp drive $[49,50]$. In this case, we can not generate the cosmological constant with ordinary massless scalar field for which the energy density is positive definite. We have the following expression for non-metricity:

$$
\nabla_{\mu} g_{\alpha \beta}=-2 g_{\mu(\alpha} \nabla_{\beta)}^{\prime} \Psi
$$

We now consider the case when both $(\Psi, \Phi)$ are present. $\tilde{C}_{\mu \nu}^{\alpha}$ is given by the following expression:

$$
\tilde{C}_{\mu \nu}^{\alpha}=\delta_{(\mu}^{\alpha} \nabla_{\nu)}^{\prime}[\ln (1+\Phi)]-\frac{1}{2} g_{\mu \nu} \nabla^{\prime \alpha}[\ln (1+\Phi)]+g_{\mu \nu} \nabla^{\prime \alpha} \Psi
$$

We obtain the following expression for the modified curvature scalar:

$$
\mathcal{R}=R^{\prime}-\frac{3}{2} \frac{1}{(1+\Phi)^{2}}\left(\nabla^{\prime} \Phi\right)^{2}+3\left(\nabla^{\prime} \Psi\right)^{2}+\frac{3}{(1+\Phi)}\left[\left(\nabla^{\prime}{ }_{\kappa} \Phi\right)\left(\nabla^{\prime \kappa} \Psi\right)\right]
$$

We have the following generalization of Einstein's equation:

$$
\begin{aligned}
\nabla_{\kappa}^{\prime}{ }_{\kappa} \nabla^{\prime \kappa}(1+\Phi) & -\frac{1}{(1+\Phi)}\left[\nabla^{\prime}(1+\Phi)\right]^{2}=0 \\
\nabla^{\prime}{ }_{\kappa} \nabla^{\prime \kappa} \Psi & =0 \\
G^{\prime}{ }_{\mu \alpha} & =8 \pi\left[P_{\mu \alpha}^{\prime}+\frac{3}{16 \pi} P^{\prime}{ }_{\mu \alpha}(\Phi)-\frac{3}{8 \pi} P_{\mu \alpha}^{\prime}(\Psi)-\frac{3}{8 \pi} P^{\prime}{ }_{\mu \alpha}(\Psi, \Phi)\right] \\
P_{\mu \alpha}^{\prime}(\Psi, \Phi) & =\frac{1}{(1+\Phi)}\left[\left(\nabla^{\prime}{ }_{(\mu} \Psi\right)\left(\nabla^{\prime}{ }_{\alpha} \Phi\right)-\frac{1}{2} g_{\mu \alpha}\left(\nabla^{\prime}{ }_{\kappa} \Psi\right)\left(\nabla^{\prime \kappa} \Phi\right)\right] \\
& \approx\left(\nabla^{\prime}{ }_{(\mu} \Psi\right)\left(\nabla^{\prime}{ }_{\alpha)} \Phi\right)-\frac{1}{2} g_{\mu \alpha}\left(\nabla^{\prime}{ }_{\kappa} \Psi\right)\left(\nabla^{\prime \kappa} \Phi\right)
\end{aligned}
$$

The first two equations are the equations for $\Phi$ and $\Psi$ and they remain same as Eqn.(45) and Eqn.(49) respectively. We find that coupling of $\Psi$ with $\Phi$ in $\mathcal{R}$ gives another contribution to source stress tensor which can be positive or negative. This is again important for dark energy research. It is interesting to note that we can always have a set of $a_{\mu \nu}$ for which only $\Phi$ is present in spacetime. On the other hand, Eq.(47) corresponds to the choice: $\Phi, E^{\alpha}{ }_{\mu \nu}=0$, in Eq.(42). It is expected that we will have non-trivial solutions of the above constraints and Eq.(47) that are in general finite when $\Psi$ is so. We will discuss this aspect after Eqn.(57). Otherwise, we can always have Eq.(47) by using the symmetric potentials introduced in the 
previous section. We can choose: $q_{\mu \nu}=\Psi(x) g_{\mu \nu}$, in Eq.(35). This will give a symmetric Ricci tensor with $\zeta=-\Psi$, in Eq. $(37)$.

We now briefly discuss the geometrical significance of $(\Psi, \Phi)$ and corresponding non-metricities given by Eqs. (46,50). We define the the non-metricity tensor by:

$$
Q_{\mu \alpha \beta}=-\nabla_{\mu} g_{\alpha \beta}=g_{\alpha \beta} \nabla_{\mu}^{\prime}[\ln (1+\Phi)] ; \quad 2 g_{\mu(\alpha} \nabla_{\beta)}^{\prime} \Psi
$$

Both $\Psi$ and $\Phi$ can be present in the manifold in a general theory. We can split $Q_{\mu \alpha \beta}$ into a trace $Q_{\mu}$ and traceless part $\bar{Q}$ in the last two indices [38]:

$$
Q_{\mu \alpha \beta}=Q_{\mu} g_{\alpha \beta}+\bar{Q}_{\mu \alpha \beta}
$$

Both trace and traceless parts of $Q_{\mu \alpha \beta}$ are finite for $\Psi$. The same is valid in general for $B^{\alpha}, E^{\alpha}{ }_{\mu \nu}$. Corresponding connections do not preserve the light cone under parallel transport and we no longer have the local Minkowski structure of spacetime [32,38]. Thus, we can not have exact $(3+1)$-splitting of the underlying manifold into space and time. We find that departure from local Minkowski geometry can give new fields like dark matter and dark energy not found ordinarily. $\bar{Q}_{\mu \alpha \beta}$ vanishes for $\Phi$. A few examples of non-metric affine connections with vanishing $\bar{Q}$, that are local gauge theories for the Weyl group (Poincare group plus dilatation), are discussed in the references given at the footnote 24 of [38]. We can have finite or vanishing torsion in such theories. These theories preserve the light cone under parallel transport and are locally Minkowski due to the reparameterization invariance of the form of the geodesic equation: $t^{\mu} \nabla_{\mu} t^{\nu}=f t^{\nu}$, where $f$ is a scalar function on the curve [28]. Here, we can use a parameterization so that: $f=\frac{1}{2} t^{\mu} \nabla^{\prime}{ }_{\mu}[\ln (1+\Phi)]$. However, this may cause problem to time-orientation when $\Phi$ is strong.

The fields $\Phi, \Psi, q_{\mu \nu}$ are purely quantum gravitational in origin. They are kinematically required in quantum gravity. In this domain, $Q_{\mu \alpha \beta}$ can not vanish due to the arguments given in Sect.III. $(\Phi, \Psi)$ can be finite everywhere and hence, they are non-localized similar to dark energy and inflation. $\Psi, q_{\mu \nu}$ break the local Minkowski structure of spacetime, and are not present in the matter-gravity coupling part of the semiclassical theory of quantum gravity which presently is locally Lorentz invariant quantum field theory in curved spaces. It is unexpected that any large coupling between $\Phi, \Psi, q_{\mu \nu}$ with ordinary matter like fermions, present in the full quantum theory, will be lost in the semiclassical limit. Thus, we assume that these fields can be present without corresponding sources from ordinary matter. However, they can couple with dark matter. They contribute non-trivially to the source stress tensor of Einstein's equation and are possible candidates for dark energy and inflation. Dark energy is non-localized, have negative pressure and is primarily observed by their gravitational effects. These make $\Psi$ a possible candidate for dark energy. $\Phi$ can be a possible candidate for inflation. The amount of dark energy is much higher than ordinary matter and $\Psi$ need not to have ordinary matter with finite hypermomentum as its sources. A complete theory of quantum gravity will illuminate this issue further. Regarding the scalar fields $(\Psi, \Phi)$, they are in line with other scalars introduced similarly, like the dilaton [51]. Observed local Lorentz invariance in many experiments indicate that $(\Psi, \Phi)$ are presently very small. This can explain the smallness of parameters like the cosmological constant required to explain present cosmological acceleration in some models. With $(\Psi, \Phi)$ being small and non-localized, their effects are usually observed in large scale phenomena and are not much significant in small scale astrophysics like that of the Solar system. This is another characteristic aspect of dark energy. However, local large inhomogeneities and anisotropies in $C^{\alpha}{ }_{\mu \nu}$ can cause significant effects on geodesic motions in the corresponding regions. Quantum fluctuations in $(\Psi, \Phi)$, including vaccuum fluctuations, can also be useful to explain different cosmological eras. When required, we can extend non-metricity and include torsion potentials to explain different cosmological observations. We can also modify the metric-affine action for this purpose. We will briefly discuss this issue at the end of this section. A related problem is to find out possible interactions and particle interpretations of fields that correspond to different representations of the Lorentz group when the local Minkowski structure of spacetime is broken strongly. This will happen in the quantum gravitational domain. This will be important to understand the relations between the complete set of fields including dark matter, dark energy, ordinary matter and gauge fields. We can continue to describe gauge theories by potentials. This is consistent with the Palatini formalism, since the potentials are analogous to connections in the geometric theory of gauge fields [52]. To illustrate, we can describe the electromagnetic field tensor by a set of potentials in a gauge invariant way, [38]:

$$
F^{\alpha \beta}=g^{\alpha \mu} g^{\beta \nu} F_{\mu \nu}=g^{\alpha \mu} g^{\beta \nu}\left[\nabla_{\mu} A_{\nu}-\nabla_{\nu} A_{\mu}+T_{\mu \nu}^{\alpha} A_{\alpha}\right]=g^{\alpha \mu} g^{\beta \nu}\left[\partial_{\mu} A_{\nu}-\partial_{\nu} A_{\mu}\right]
$$

The above definition is valid in the metric-affine theory with finite torsion. $T_{\mu \nu}^{\alpha}$ vanish in the Palatini formalism. We find that $A_{\mu}$ do not couple with $C_{\mu \nu}^{\alpha}$. This aspect remains valid for the non-Abelian gauge 
theories where the field-strength tensors are given by gauge-covariant curls of the corresponding potentials [53]. The discussions above Eq.(56) are new perspectives in quantum gravity deduced mathematically in this article and partly supported by cosmological observations.

We can try to introduce coupling between $(\Psi, \Phi)$ and fermions in quantum gravity by removing the antisymmetry and metric compatibility conditions on contorsion provided the non-local character of $(\Psi, \Phi)$ is maintained. We will also have to explain why such coupling is not significant in the semiclassical and classical theories. Otherwise, we can have observable effects of non-metricity in events like NS-NS, NS-WD and WD-WD mergers $[54,55]$.

As a first approximation, we can use semiclassical and classical theories like quantum and classical fields in curved spaces to find the effects of $(\Psi, \Phi)$ when the full quantum theory is not much significant. This will be useful in cosmic epoch. Lastly, the action for scalar-tensor theories is given by [6]:

$$
S=\int d^{4} x \sqrt{-g}\left[f\left(\phi, R^{\prime}\right)-\zeta(\phi)\left(\nabla^{\prime} \phi\right)^{2}\right]+S_{m}\left(\psi, g_{\mu \nu}\right)
$$

Where, $\psi$ represents other matters including radiation. We find from Eqs. $(44,48)$ that the fields $(\Psi, \Phi)$ can be described by such theories with $\zeta(\Psi)=-3$ and $\zeta(\Phi)=\frac{3}{2} \frac{1}{(1+\Phi)^{2}} . f\left(\phi, R^{\prime}\right)=R^{\prime}$ for both fields. This indicates some of the quintessence, k-essence scalars can have purely geometrical origin similar to $(\Psi, \Phi)$.

Here we consider $E_{\mu \nu}^{\mu}$. In the following we will use: $\Omega=\ln (1+\Phi)$ which simplify the calculations. We first note that we can define $\tilde{C}_{\mu \nu}^{\alpha}$ of Eqn.(41) in the following alternate form by extracting the trace part in the first two indices of $D_{\mu \nu}^{\alpha}$ :

$$
\tilde{C}_{\mu \nu}^{\alpha}=\delta_{(\mu}^{\alpha} \nabla^{\prime}{ }_{\nu)} \Omega-\frac{1}{2} g_{\mu \nu} \nabla^{\prime \alpha} \Omega+2 \delta_{(\mu}^{\alpha} \nabla^{\prime}{ }_{\nu)} \Xi+L_{\mu \nu}^{\alpha}
$$

where $L_{\mu \nu}^{\mu}=0$. It is easy to find that $\Xi$ has properties similar to $\Psi$ and contributes a negative stress-tensor. We now consider $E^{\alpha}{ }_{\mu \nu}$ of Eqn.(42). $E^{\alpha}{ }_{\mu \nu}$ is traceless in the lower indices and symmetry of the Ricci tensor indicates that: $E_{\alpha \nu}^{\alpha}=\nabla^{\prime}{ }_{\nu} \zeta$ where $\zeta$ is a scalar that we choose to vanish since we have already extracted two scalars. As before, we simplify the treatment by introducing a traceless symmetric second rank tensor $l_{\mu \nu}$ that is divergenceless in either of its indices and define: $E_{\mu \nu}^{\alpha}=\nabla^{\alpha} l_{\mu \nu}$. This gives: $E_{\kappa \lambda}^{\kappa}=0$. The modified curvature scalar is now given by:

$$
\begin{aligned}
\mathcal{R} & =R^{\prime}-\frac{3}{2}\left(\nabla^{\prime} \Omega\right)^{2}+3\left(\nabla^{\prime} \Psi\right)^{2}+3\left[\left(\nabla^{\prime}{ }_{\kappa} \Omega\right)\left(\nabla^{\prime \kappa} \Psi\right)\right] \\
& -R_{\alpha \mu \beta \nu}^{\prime} l^{\alpha \beta} l^{\mu \nu}+R_{\alpha \mu}^{\prime} l^{\alpha \lambda} l_{\lambda}^{\mu}
\end{aligned}
$$

where the primed quantities are evaluated using the Levi-Civita connections, $l_{\mu \nu}=l_{\nu \mu}$ and we have used the following constraints:

$$
l_{\mu}^{\mu}=0, \nabla^{\prime \mu} l_{\mu \nu}=0
$$

Thus, we have replaced $a_{\mu \nu}$ introduced in Eqns. $(11,12)$ by the set of three fields $\left(\Phi, \Psi, l_{\mu \nu}\right)$ that describe two scalar bosons and one spin-2 boson. The configuration space is now described by the four fields: $\left(g_{\alpha \beta}, \Phi, \Psi, l_{\mu \nu}\right)$. $\mathcal{R}$ does not contain derivatives of $l_{\mu \nu}$ and there is no coupling between them and $(\Phi, \Psi)$. We have: $\frac{\delta \mathcal{R}}{\delta\left(l^{\kappa \mu}\right)}=2\left(R_{(\kappa|\alpha|}^{\prime} l_{\mu)}^{\alpha}-R_{\kappa \lambda \mu \alpha}^{\prime} l^{\lambda \alpha}\right)$. We can use Lagrange's multipliers to choose $l_{\mu \nu}=0$ as a valid solution which corresponds to a theory with only $(\Phi, \Psi)$ finite as we have considered in this article. Again, we can always consider $a_{\mu \nu}, q_{\mu \nu}$ to be given by $\Phi(x) g_{\mu \nu}, \Psi(x) g_{\mu \nu}$ and consider a theory with only $(\Phi, \Psi)$ finite. When required, we can add suitable $\left(\Phi, \Psi, l_{\mu \nu}\right)$ dependent terms in Eqn.(59) including potential terms, terms that can lead to dynamical $l_{\mu \nu}$ and mass terms. We then have:

$$
\mathcal{L}=\mathcal{R}-\mathcal{V}
$$

where $\mathcal{V}$ contains the added terms which should be consistent with: $\nabla^{\prime \alpha} G^{\prime}{ }_{\mu \alpha}=0$, if we express the equations in the form similar to Eqn.(45). The connections in Eqn.(42) are to be evaluated using the solutions obtained from the above Lagragian density. We now consider the case when only $\Phi$ and $\Psi$ are present. In general, the above Lagrangian will give coupled equations for $\Phi$ and $\Psi$ that can lead to interesting effects. We can construct a simple theory by choosing $\mathcal{V}=\mathcal{V}_{1}(\Phi)+\mathcal{V}_{2}(\Psi)+3\left[\left(\nabla^{\prime}{ }_{\kappa} \Omega\right)\left(\nabla^{\prime \kappa} \Psi\right)\right]$. This gives a set of two decoupled equations for $\Phi$ and $\Psi$. 


\section{Conclusion}

In this article, we have considered the issue of construction of covariant derivative operator in quantum gravity. We have used the canonical quantization approach and Palatini action to illustrate this issue. We have found it is more perceptive to use all basic covariant structures of geometry to formulate a quantum theory of gravity. This is valid irrespective of the presence and nature of sources. These covariant structures of geometry include metric and a third rank tensor $C_{\mu \nu}^{\alpha}$. We call this field as supertorsion. The later field leads to affine connections more general than the metric compatible Levi-Civita connections. Symmetric part of $C_{\mu \nu}^{\alpha}$ in the lower indices can introduce scalar fields and symmetric second rank covariant tensors. Antisymmetric part of this tensor in the lower indices gives half of torsion tensor.

We have found that the familiar Palatini formalism and metric-affine gravity are not sufficient to construct a quantum theory of gravity. We have considered possible extensions of these formalisms to construct a quantum theory. We can do so by using potentials to express connections. Alternatively, we can use more general actions than the Palatini action to construct a quantum theory. We can include higher order curvature inavariants like $R_{\mu \nu} R^{\mu \nu}$ in the metric-affine action. We can also use the Palatini $f(R)$ gravity or metric-affine $f(R)$ gravity. In the simple cases considered in this article, the potential formalism introduce two massless scalar fields in the theory. They are non-localized and one of them contribute negative source stress-tensor to the familiar Einstein equation. This can be useful to explain early universe inflation and dark energy that require negative pressure. This is also important for wormholes and warp drive which require negative energy. The other scalar field, when supplemented by suitable potentials, will be useful to explain the early universe inflation [56,57]. We can introduce other fields from general affine connections. We have found that general affine connections do not preserve the light cone under parallel transport and bring us beyond a strict local Minkowski spacetime. Inexactness of parallel transport in quantum gravity mentioned below Eq.(16) also does not preserve the light cone under parallel transport. Inflation, dark energy, dark matter, renormalizability and the issue of $(3+1)$-splitting of spacetime into space and time have been primary fields of quantum gravity research that have found a common place here. We find that the Lagrangian formalism, where we do not need $(3+1)$-splitting of spacetime into space and time, is more general to construct a quantum theory of gravity $[28,30,58]$. Affine connections can also be useful to construct theories alternatives of cosmic inflation [59].

\section{Acknowledgement}

I am thankful to the reviewers for a few improvements.

\section{Appendix:A}

Here, we make a few comments on what will happen to Eq.(20) when we choose a different operator ordering in the Levi-Civita connections than that discussed below Eq.(16). We first discuss what happens to the dicussions below Eq.(20) when we consider a symmetric ordering in the Levi-Civita symbol. The r.h.s of Eq.(20) is now non-vanishing and we get the following condition when we consider the action of $\hat{\nabla}_{x k}^{\prime}$ on both sides of Eq.(19):

$$
-\left[\left[\hat{g}^{\alpha \tau}(t, \vec{x}), \hat{M}_{\tau k(p}(t, \vec{x})\right] \hat{g}_{|\alpha| l)}(t, \vec{x}), \hat{\pi}^{p l}(t, \vec{y})\right]=i \hat{\nabla}_{x k}^{\prime}\left[\delta_{(p}^{p} \delta_{l)}^{l} \delta(\vec{x}, \vec{y})\right]
$$

Where, $M_{\alpha \mu \nu}=\frac{1}{2}\left[\partial_{\mu}\left(g_{\alpha \nu}\right)+\partial_{\nu}\left(g_{\mu \alpha}\right)-\partial_{\alpha}\left(g_{\mu \nu}\right)\right]$ and we have kept $\hat{g}_{\alpha l}$ and $\hat{g}_{\alpha p}$ at the right of the connections. We have used the fact that $\hat{\nabla}_{\mu}^{\prime}\left[\hat{g}_{\alpha \beta}\right] \equiv 0$, when the operator ordering in the Levi-Civita connections is taken to be the same as that discussed below Eq.(16) and given by Eq.(7). We also have, $\hat{g}^{\alpha \kappa} \hat{g}_{\kappa \beta}=\delta_{\beta}^{\alpha}$. We need to solve the constraints and gauge fixing conditions to find exact expressions of both sides. We can construct a general form as follows. The r.h.s was mentioned below Eq.(19). The commutator within the commutator in the l.h.s of the above equation, when finite, will be independent of $\vec{y}$ and will contain singular quantities in coordinates $\vec{x}$. If we multiply both sides by a regular function $f(\vec{y})$ and integrate w.r.t $\vec{y}$ over the spatial section, the r.h.s will give a regular expression although the l.h.s remains divergent. In the most favorable situation, the l.h.s can be of the form: $i s(\vec{x}, \vec{x}) \hat{\nabla}_{x k}^{\prime}\left[\delta_{(p}^{p} \delta_{l)}^{l} \delta(\vec{x}, \vec{y})\right]$, where $s(\vec{x}, \vec{x})$ is a singular quantity. This is not same as the r.h.s. Thus, we again obtain a contradiction similar to that discussed below Eq.(20). Lastly, many other ordering of $\partial_{\lambda} g_{\alpha \beta}$ and $g^{\mu \nu}$ in the Levi-Civita connections can be expressed as a linear 
combination of the ordering given by Eq.(7) and the symmetric ordering considered here. Corresponding

covariant derivative is given as: $\hat{\nabla}_{\mu}=m \hat{\nabla}_{1 \mu}+(1-m) \hat{\nabla}_{2 \mu}$, where the covariant derivatives in the r.h.s correspond to the two orderings mentioned before and $m$ can be negative. With $\hat{\nabla}_{1 \mu}\left(\hat{g}_{\alpha \beta}\right)=0$, we will again have contradictions similar to those discussed in this article.

\section{References}

[1] A. A. Starobinisky, Phys. Lett. B 91, 99 (1980).

[2] A. H. Guth, Phys. Rev. D 23, 347 (1981).

[3] D.N. Spergel et al [WMAP Collaboration], Astrophys. J. Suppl. 148, 175 (2003).

[4] D.N. Spergel et al [WMAP Collaboration], Astrophys. J. Suppl. 170, 377 (2007).

[5] E. Komatsu et al [WMAP Collaboration], Astrophys. J. Suppl. 180, 330 (2009).

[6] L. Amendola and S. Tsujikawa, Dark Energy, (Cambridge University Press, Cambridge, 2010).

[7] F. Zwicky, Helv. Phys. Acta 6, 110 (1933).

[8] A. De Felice and S. Tsujikawa, Living Reviews in Relativity. 13 (2010).

[9] D. N. Vollick, Phys. Rev. D 68, 063510 (2003).

[10] S. Weinberg, The Cosmological Constant Problem, Rev. Mod. Phys. 61, 1 (1989).

[11] Y. Fujii, Phys. Rev. D 26, 2580 (1982).

[12] T. Chiba, T. Okabe and M. Yamaguchi, Phys. Rev. D 62, 023511 (2000).

[13] A. Y. Kamenshchik, U. Moschella and V. Pasquier, Phys. Lett. B 511, 265 (2001).

[14] S. Capozzillo, Int. J. Mod. Phys. D 11, 483 (2002).

[15] L. Amendola, Phys. Rev. D 60, 043501 (1999).

[16] J. P. Uzan, Phys. Rev. D 59, 123510 (1999).

[17] G. R. Dvali, G. Gabadadze and M. Porrati, Phys. Lett. B 485, 208 (2000).

[18] V. Sahni and Y. Shtanov, JCAP 311, 014 (2003).

[19] R. Utiyama and B. S. DeWitt, J. Math. Phys. 3, 608 (1962).

[20] K. S. Stelle, Phys. Rev. D 16, 953 (1977).

[21] B. S. DeWitt, Phys. Reports 19, No.6 (1975).

$[22]$ N. D. Birrel and P. C. W. Davies, Quantum Fields in Curved Space (Cambridge University Press, Cambridge, 1982).

[23] I. L. Buchbinder, S. D. Odintsov, and I. L. Shapiro, Effective Actions in Quantum Gravity (IOP Publishing, Bristol, 1992).

[24] G. A. Vilkovisky, Class. Quant. Grav. 9, 895 (1992).

[25] J. G. Hocking and G. S. Young, Topology (Dover Publications, Inc., New York, 1961).

[26] D. Lovelock and H. Rund; Tensors, Differential Forms, and Variational Principals (Dover Publications, Inc., New York, 1989).

[27] L. D. Landau and E. M. Lifshitz; The Classical Theory of Fields (Butterworth-Heinenann, Oxford, 1998).

[28] R. M. Wald, General Relativity (The University of Chicago Press, Chicago and London, 1984).

[29] C. W. Misner, K. S. Thorne and J. A. Wheeler, Gravitation (W.H. Freeman and company, New York, 1970).

[30] S. W. Hawking: The Path-Integral Approach to Quantum Gravity in S. W. Hawking and W. Israel, eds, General Relativity: An Einstein Centenary Survey (Cambridge University Press, 1979).

[31] A. Ashtekar, Lectures on Non-Perturbative Canonical Gravity (World Scientific, Singapore, 1991).

[32] F. W. Hehl et al, Phys. Rept. 258, 1 (1995).

[33] D. Iosifidis, Class. Quant. Grav. 36, 8 (2019).

[34] M. Ferraris, M. Francavigilia and C. Reina, Gen. Rel. Grav. 14, 243-254, (1982).

[35] V. Moncrief, J. Math. Phys. 16, 493.

[36] C. Isham: Canonical Quantum Gravity and the Question of Time, in J. Ehlers and H. Friedrich, eds, Canonical Gravity: From Classical to Quantum; Proceeding of the 117th WE Heraeus Seminar Held at Bad Honnef, Germany,1993 (Springer-Verlag, 1994).

[37] K. Sundermeyer, Constrained Dynamics (Springer-Verlag, 1995).

[38] F. W. Hehl et al, Rev. Mod. Phys. 48 (1976) 3641.

[39] F. De Felice and C.J.S Clarke, Relativity on Curved Manifolds (Cambridge University Press, Cambridge, 1990).

[40] C. Itzykson and J. B. Zuber, Quantum Field Theory (Dover Publications, Inc. Mineola, 2005)

[41] P. Ramond, Field Theory: A Modern Primer (Addison Wesley, 1990).

[42] R. T. Hammond, Rep. Prog. Phys. 65, 599 (2002). 
[43] N. Koloper, Phys. Rev. D 44, 2380 (1991).

[44] A. Golovnev, M. Mukhanov and V. Manchurin, JCAP 0806:009, 2008.

[45] R. Emami et al, JCAPO3 (2017) 058.

[46] N. Bartolo et al, Phys. Rev. D 97, 023503 (2018).

[47] D.E. Nevill, Phys.Rev. 23D (1981) 1244; 25D (1982) 573.

[48] E. Sezgin and P. van Nieuwenhuizen, Phys. Rev. 21D (1981) 3269.

[49] J. V. Narlikar, Pramana 2(3): 158-170 (1974).

[50] L. H. Ford and T. A. Roman, Scientific American 28246 (2000)

[51] M. Gasperini and G. Veneziano, Phys. Rept. 373, 1 (2003).

[52] M. Nakahara, Geometry, Topology and Physics (Adam Hilger, Bristol and New York, 1990).

[53] S. Weinberg, The Quantum Theory of Fields, Vol.II (Cambridge University Press, Cambridge, 1996).

[54] B. P. Abbott et al, Phys. Rev. Lett. 119, 161101, (2017).

[55] B. P. Abbott et al, Astroph. J. 848, L13, (2017). [1710.05834].

[56] A. R. Liddle and D. H. Lyth, Cosmological Inflation and Large Scale Structures (Cambridge University Press, 2000).

[57] J. Martin, C. Ringeval and V. Vennin, Physics of the Dark Universe, 5-6, 75 - 235, (2014).

[58] K. Ghosh, https://hal.archives-ouvertes.fr/hal-02105422

[59] N. J. Poplawski, Phys. Lett. B 694, 181 (2010). 